\title{
Inhibition de différentes protéinases fongiques dans le lait cru de plusieurs espèces de ruminants
}

\author{
par \\ G. HUMBERT, S.E. CHUON, L. CASABIANCA et C. ALAIS
}

\section{Ré s u m é}

Le lait cru de vache, de brebis et de chèvre renferme un inhibiteur de la protéinase acide de Mucor miehei. L'inhibition est beaucoup moins marquée pour la protéinase acide de Mucor pusillus; elle est nulle pour celle de Endothia parasitica et pour les protéinases gastriques (chymosine et pepsine bovines). L'inhibition a été constatée dans le lait de vache pour une protéinase neutre de Bacillus subtilis.

L'activité inhibitrice paraît constante dans le temps chez une même vache, mais elle varie beaucoup d'un individu à l'autre. Elle est détruite par les traitements thermiques de type pasteurisation, mais pas aux températures de congélation. Elle se localise dans le lactosérum et elle n'est pas dialysable ; l'inhibiteur doit être de nature protéique.

L'inhibition concerne aussi bien la protéolyse primaire que la coagulation proprement dite.

Mots clés

Protéinases fongiques, coagulation, protéolyse, lait, vache, chèvre, brebis.

Titre abrégé

Inhibition de protéinases fongiques dans le lait cru.

Laboratoire de Biochimie Appliquée, Université de Nancy I - 54506 Vandœuvreles-Nancy. 


\section{S u m m a ry}

\section{INHIBITION OF DIFFERENT FUNGAL PROTEINASES IN RAW MILK OF SEVERAL RUMINANT SPECIES}

The inhibition of the milk clotting activity of the Mucor miehei acid proteinase, which was already observed in bovine milk, is found also in sheep's and goat's milk. It is lower for the Mucor pusillus acid proteinase in these three milks. A Bacillus subtilis neutral proteinase is also inhibited in cow's milk. There is no inhibition for the Endothia parasitica acid proteinase and the bovine gastric proteinases (chymosin and pepsin).

Most of these studies were performed with the Mucor miehei proteinase. The inhibition seems to be constant for each cow but there are large individual variations.

The inhibition of the clotting activity is suppressed by pasteurization $\left(73^{\circ} \mathrm{C}, 25 \mathrm{~s}\right)$ but not at freezing temperatures. The inhibitor is probably a protein fraction; it is localized in the acid whey and the supernatant of milk ultracentrifugation. It is not dialysable. The inhibition in cow's milk is approximatively proportional to the inhibitor concentration.

The inhibition concerns both proteolysis and coagulation itself in cow's and goat's milk. The release of the non-protein nitrogenous substances is notably hindered during the "primary reaction".

Key words

Fungal proteinases, coagulation, proteolysis, milk, cow, goat, sheep.

Run title

Inhibition of fungal proteinases in raw milk.

\section{INTRODUCTION}

L'activité coagulante de la protéinase de Mucor miehei est sensible à une substance inhibitrice contenue dans la fraction " protéines solubles " du lait de vache cru (Alais, Ducroo et Delecourt, 1974). La proportionnalité entre le temps de coagulation et la dilution de la solution d'enzyme n'existe plus; au-dessus d'une certaine dilution, l'allongement du temps de coagulation est trop grand. L'inhibition disparaît dans les conditions de la pasteurisation; elle persiste après " thermisation »; elle pose des problèmes dans certaines fabrications de fromage (Ramet et Alais, 1973).

La chymosine ne subit pas cette inhibition; par contre, on sait qu'elle est sensible à des substances globuliniques du sang (Briot, 
1900 ; Tsugo et Yamauchi, 1953; Singh et Ganguli, 1973) ou du lait et du colostrum de vache (Puhan, 1966, 1967).

En poursuivant l'étude du phénomène d'inhibition, nous avons cherché à savoir d'une part, si elle se retrouve dans le lait d'autres espèces communes de ruminants et, d'autre part, si l'inhibition concerne aussi l'activité protéolytique (réaction primaire). De plus, nous avons étudié les effets de divers traitements sur l'acivité inhibitrice du lait de vache.

\section{MATERIEL ET METHODES}

\section{Laits}

Le lait de vache provient de deux élevages de la région de Nancy; le lait de chèvre provient de la ferme de l'ENSAIA et le lait de brebis, d'un élevage savoyard.

Le lait reconstitué standard (dépourvu d'activité inhibitrice) est obtenu à partir d'une poudre maigre « low heat » (Lesaffre, Cérences) : on dissout $12 \mathrm{~g}$ de poudre dans $100 \mathrm{ml}$ d'une solution $0,003 \mathrm{M}$ de chlorure de calcium, $\mathrm{pH}$ 6,5. Dans certaines expériences, le lait est reconstitué dans le surnageant d'ultracentrifugation (64000 g $\times 45 \mathrm{~min})$ d'un lait cru. Le lait reconstitué est laissé au repos à $20^{\circ} \mathrm{C}$ pendant $1 \mathrm{~h}$ à l'obscurité pour que les équilibres ioniques soient établis.

\section{Enzymes coagulantes}

Nous avons utilisé 5 préparations de protéinases fongiques :

- protéinase de Mucor miehei (MmR), souche NRRL 3169, fournie par la Sté Rapidase (Seclin);

- protéinase de Mucor miehei (MmN) souche CBS 37065, fournie par Novo-Industrie (Danemark);

- protéinase de Mucor pusillus (Mp) souche Lindt F 27, fournie par Meito Sangyo (Japon);

- protéinase de Endothia parasitica (Ep) souche ATCC 14729, fournie par Pfizer Co (U.S.A.);

- protéinase de Bacillus subtilis (Bs) préparée au laboratoire (Alais et Novak, 1968).

En outre, nous avons utilisé pour certaines comparaisons la pepsine bovine (EC 3.4.23.1) et la chymosine bovine (EC 3.4.23.4) fournies par les Ets Hansen (Danemark).

Chaque enzyme est dissoute dans le tampon phosphate 0,05 M, $\mathrm{pH}$ 6,5. La dilution « 1 » est celle qui nous donne dans les conditions précisées au paragraphe suivant, un temps de coagulation de 3 à 
4 min du lait reconstitué; la dilution maximale donne un temps de $60 \mathrm{~min}$ environ (au-delà, la mesure est imprécise).

\section{Méthodes}

- Le temps de coagulation (tc) est mesuré, selon Berridge (1945) en observant un film de lait s'écoulant sur la paroi d'un tube en verre incliné en rotation dans un bain-marie à $30^{\circ} \mathrm{C}$. Le tube contient $10 \mathrm{ml}$ de lait et $0,5 \mathrm{ml}$ de solution d'enzyme.

- En ce qui concerne les laits de vache individuels, dans un but de standardisation, on a séparé de chaque lait le surnageant d'ultracentrifugation, qui renferme, on le sait, le ou les facteurs d'inhibition et on a reconstitué dans ces surnageants un même lait en poudre (exempt d'inhibiteur). On a donc le même phospho-caséinate de calcium dans tous les cas et le même $\mathrm{pH}(6,5)$.

- La protéolyse a été mesurée à $37^{\circ} \mathrm{C}$ et pH 6,5 par l'augmentation de l'azote non protéique (NPN) soluble dans $12 \%$ d'acide trichloracétique $(\mathrm{p}: \mathrm{v})$ :

- dans le cas de la caséine entière, on a fait réagir l'enzyme $\mathrm{MmR}$ sur une solution à $1 \%$ de caséine contenant 1 ou $2 \%$ de lactosérum séché par lyophilisation après dialyse, soit préalablement chauffé à $73^{\circ} \mathrm{C}, 25 \mathrm{~s}$ (c'est l'essai témoin), soit naturel, c'est-à-dire conservant l'activité inhibitrice;

- dans le cas du lait, on a fait réagir la même enzyme à différentes concentrations dans un lait de mélange de vache et dans un lait de mélange de chèvre.

\section{RESULTATS}

\section{Inhibition de l'activité coagulante}

1. Lait de différentes espèces et enzymes de différentes origines

Les essais de coagulation ont été faits avec des laits de mélange de vache, de chèvre et de brebis, et différentes dilutions de 7 enzymes coagulantes. Les courbes reliant le temps de coagulation à la dilution de l'enzyme sont représentées sur les figures 1,2 et 3 . On observe que :

- la chymosine, la pepsine bovine et la protéinase de Endothia parasitica donnent des représentations linéaires avec tous les laits, qu'ils soient crus ou chauffés. Il n'y a pas d'inhibition apparente;

- les protéinases des Mucor sont sensibles à un inhibiteur présent dans les laits crus des 3 espèces. L'inhibition disparaît après chauffage de ces trois laits. Avec la protéinase de Mucor pusillus l'inhibition semble moins marquée qu'avec les autres enzymes; les 


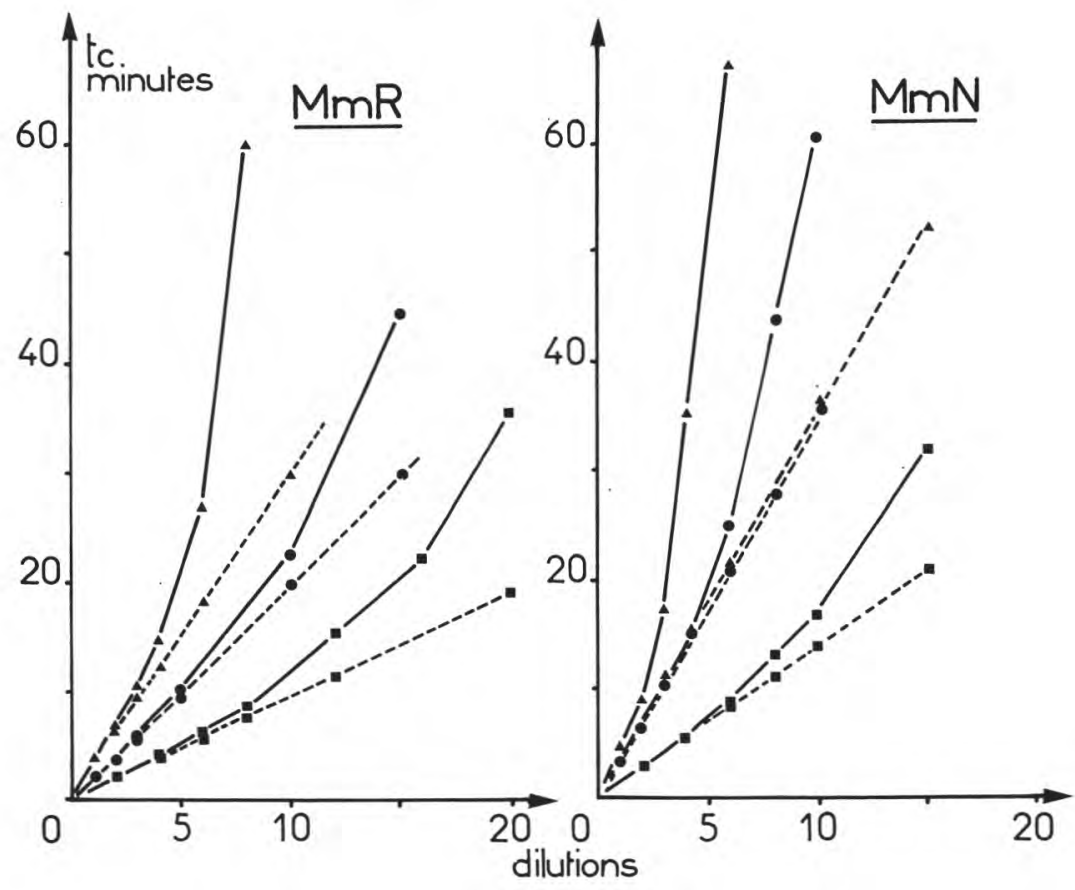

Fig. 1

Evolution du temps de coagulation des laits de vache, de brebis et de chèvre en fonction de la dilution des protéinases de Mucor miehei ( $\mathrm{MmR}$ et $\mathrm{MmN}$ ) ;

lait de vache cru

lait de brebis cru

$\Delta$ et chauffé $\mathbf{\Delta}--\mathbf{A}\left(73^{\circ} \mathrm{C}, 25 \mathrm{~s}\right)$

$\bullet-$ et chauffé $\bullet-\cdots\left(73^{\circ} \mathrm{C}, 25 \mathrm{~s}\right)$

lait de chèvre cru m et chauffé $1-\ldots\left(73^{\circ} \mathrm{C}, 25 \mathrm{~s}\right)$

Les dilutions 1 des enzymes coagulent le lait cru en $3-4 \min$ à $30^{\circ} \mathrm{C}$.

Evolution of clotting time of cows sheeps and goat's milk in function of the dilution of Mucor miehei proteinases ( $M m R$ and $M m N)$;

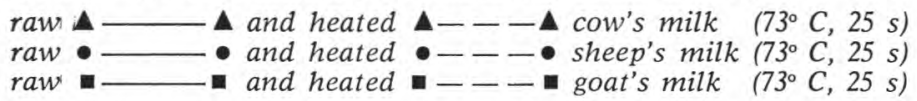

The enzymes dilution 1 clots the raw milks in 3-4 min at $30^{\circ} \mathrm{C}$.

courbes tracées à partir du lait cru s'écartent peu des courbes correspondantes au lait chauffé $\left(73^{\circ} \mathrm{C}, 25 \mathrm{~s}\right)$. La protéinase de Bacillus subtilis est sensible à l'inhibiteur présent dans le lait de vache.

\section{Laits de vache individuels}

On a utilisé l'enzyme MmR et l'on est parti de douze laits individuels de vaches en pleine lactation. La figure 4 présente les courbes obtenues avec six laits; les autres laits ont donné des courbes se superposant approximativement aux précédentes. On voit qu'il existe 


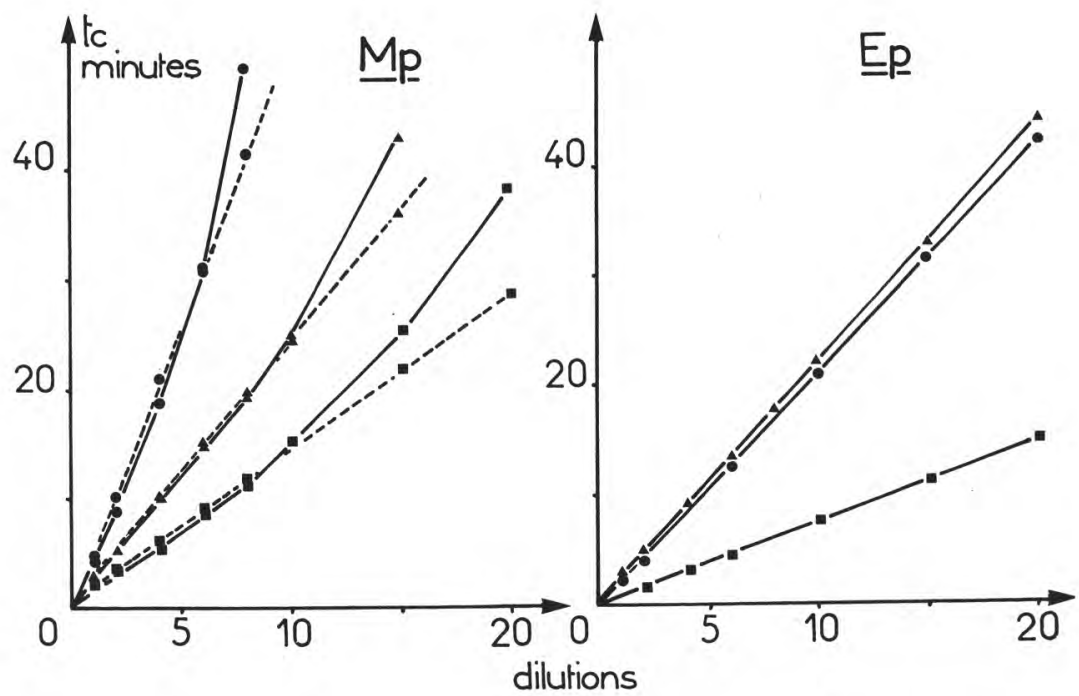

Fig. 2

Evolution du temps de coagulation du lait de vache, de brebis et de chèvre en fonction de la dilution des protéinases de Mucor pusillus (Mp) et Endothia parasitica (Ep) (voir légende fig. 1).

Evolution of clotting time of cow's, sheep's and goat's milks in function of the dilution of Mucor pusillus $(M p)$ and Endothia parasitica (Ep) proteinases (see legend fig. 1).

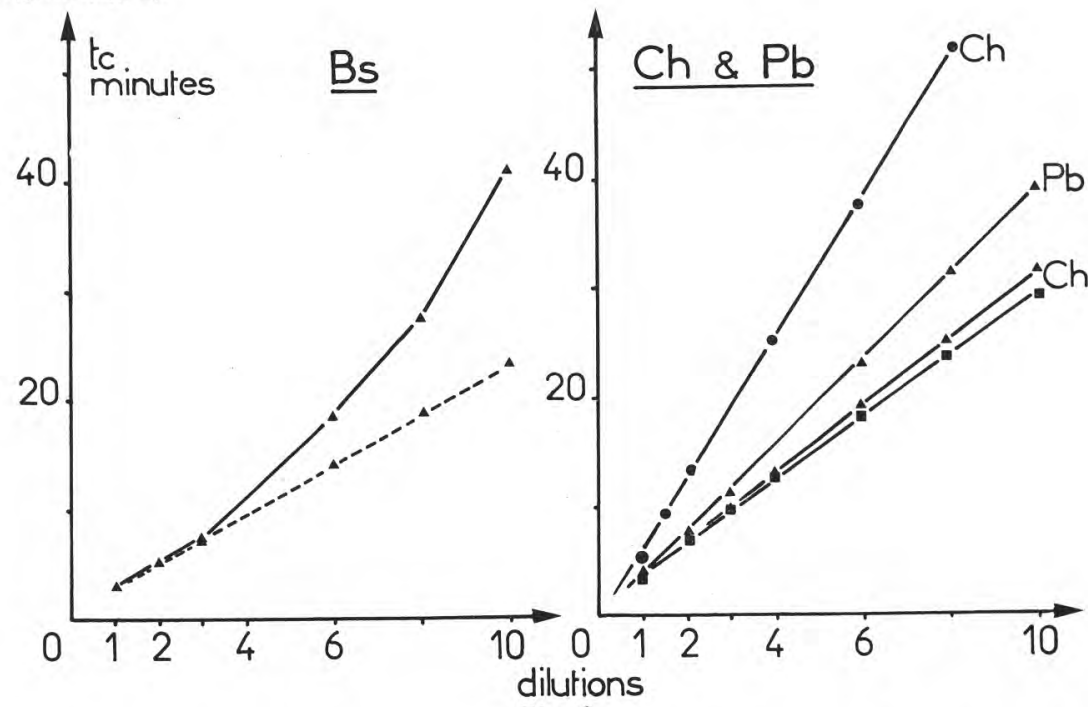

Fig. 3

Evolution du temps de coagulation du lait de vache, de brebis et de chèvre en fonction de la dilution de la chymosine $(\mathrm{Ch})$, de la pepsine bovine $(\mathrm{Pb})$ et de la protéinase de Bacillus subtilis (Bs) (voir légende fig. 1).

Evolution of clotting time of cow's, sheep's and goat's in function of the dilution of rennin (Ch), of bovine pepsine $(\mathrm{Pb})$ and of Bacillus subtilis (Bs) proteinase (see legend fig. 1). 


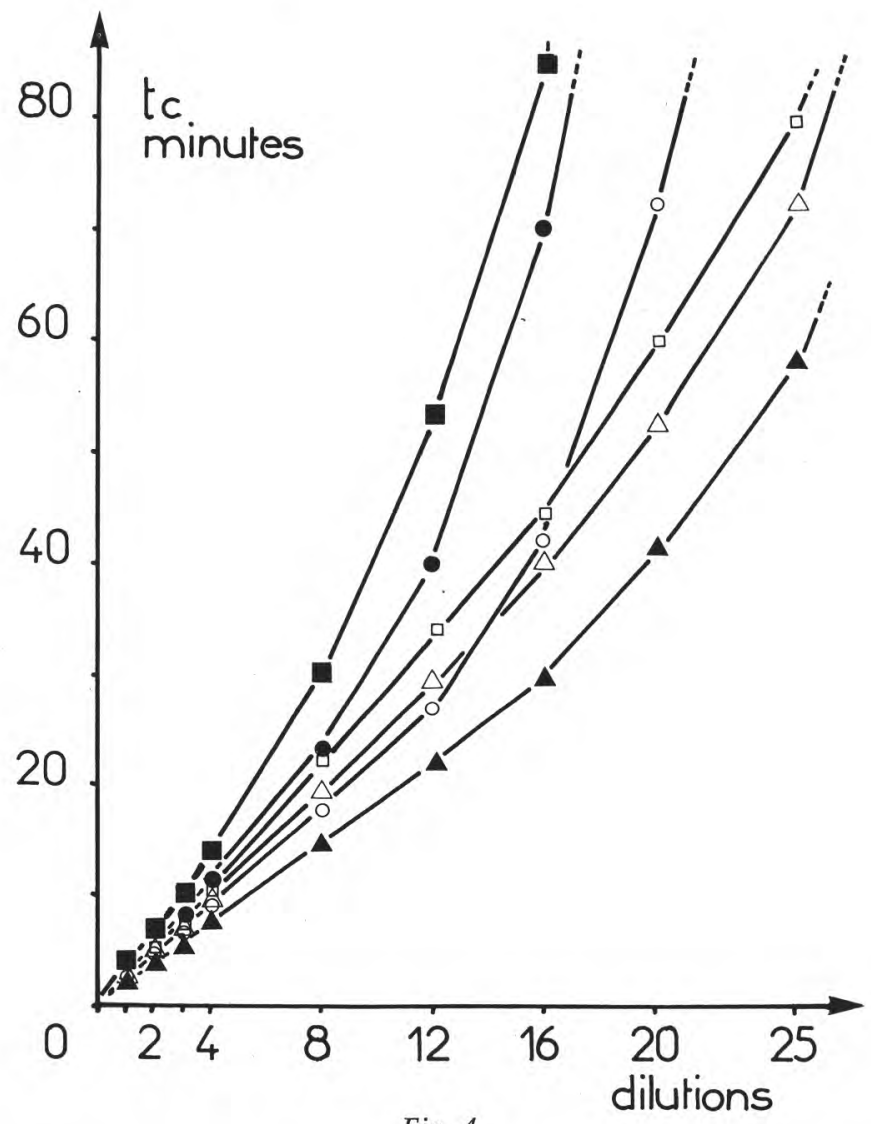

Fig. 4

Evolution du temps de coagulation de laits crus individuels de vache en fonction de la dilution de la protéinase MmR (Concentration en enzyme dans le lait pour la dilution $1: 0,04 \% ; 30^{\circ} \mathrm{C}$ $\mathrm{pH} 6,5)$.

Evolution of clotting-time of individual bovine raw milks in function of the dilution of the MmR-proteinase (enzyme concentration in milk for dilution $\left.n^{\circ} 1: 0,04 \% ; 30^{\circ} \mathrm{C}-\mathrm{pH} 6,5\right)$.

des différences sensibles d'un lait à l'autre. La dilution au-delà de laquelle le temps de coagulation n'est plus proportionnel à la dilution de l'enzyme varie de 4 à 16 (la solution initiale coagule le lait en 3-4 $\mathrm{min})$.

3. Lait d'une même vache plusieurs fois dans le temps

Nous avons effectué des épreuves de coagulation avec l'enzyme $\mathrm{MmR}$ à plusieurs dilutions, en prenant le lait de trois vaches et 


\section{TABLEAU 1 - TABLE 1}

Evolution de l'activité inhibitrice au cours du temps (tc lait $\mathrm{cru} / \mathrm{tc}$ lait chauffé ; concentration en enzyme dans le lait pour la dilution $\left.1: 0,04 \%, 30^{\circ} \mathrm{C}, \mathrm{pH} 6,5\right)$

Evolution of inhibitory activity in function of time (Ct raw milk/Ct heated milk; Enzyme concentration in milk for dilution $\left.n^{\circ} 1: 0,04 \%, 30^{\circ} \mathrm{C}, \mathrm{pH} 6,5\right)$

\begin{tabular}{|c|c|c|c|c|}
\hline \multirow[b]{3}{*}{$\begin{array}{r}\text { Vache } n^{\circ} 1 \\
\text { Jour } \mathrm{J} \\
\mathrm{J}+3 \\
\mathrm{~J}+8 \\
\mathrm{~J}+18 \\
\mathrm{~J}+21 \\
\mathrm{~J}+23\end{array}$} & \multicolumn{4}{|c|}{ Dilution de la solution d'enzyme } \\
\hline & x 8 & $\mathrm{x} 12$ & x 16 & x 20 \\
\hline & $\begin{array}{l}1,04 \\
1,0 \\
1,0 \\
1,02 \\
1,09 \\
1,01\end{array}$ & $\begin{array}{l}1,11 \\
1,10 \\
1,09 \\
1,05 \\
1,19 \\
1,16\end{array}$ & $\begin{array}{l}1,33 \\
1,15 \\
1,14 \\
1,11 \\
1,31 \\
1,23\end{array}$ & $\begin{array}{l}1,39 \\
1,30 \\
1,19 \\
1,15 \\
1,40 \\
1,64\end{array}$ \\
\hline $\begin{array}{r}\text { Vache } n^{\circ} 2 \\
\mathrm{~J} \\
\mathrm{~J}+3 \\
\mathrm{~J}+8 \\
\mathrm{~J}+18 \\
\mathrm{~J}+21 \\
\mathrm{~J}+23\end{array}$ & $\begin{array}{l}1,03 \\
1,04 \\
1,04 \\
1,14 \\
1,15 \\
1,09\end{array}$ & $\begin{array}{l}1,14 \\
1,15 \\
1,12 \\
1,35 \\
1,33 \\
1,19\end{array}$ & $\begin{array}{l}1,26 \\
1,35 \\
1,23 \\
1,47 \\
1,41 \\
1,30\end{array}$ & $\begin{array}{l}1,30 \\
1,55 \\
1,40 \\
1,67 \\
\overline{1,48}\end{array}$ \\
\hline $\begin{array}{r}\text { Vache } n^{\circ} 3 \\
\mathrm{~J} \\
\mathrm{~J}+3 \\
\mathrm{~J}+8 \\
\mathrm{~J}+18 \\
\mathrm{~J}+21 \\
\mathrm{~J}+23\end{array}$ & $\begin{array}{l}1,10 \\
1,0 \\
1,09 \\
1,11 \\
1,15 \\
1,17\end{array}$ & $\begin{array}{l}1,22 \\
1,12 \\
1,15 \\
1,15 \\
1,28 \\
1,32\end{array}$ & $\begin{array}{l}1,40 \\
1,35 \\
1,35 \\
1,35 \\
1,40 \\
1,48\end{array}$ & $\begin{array}{l}1,58 \\
1,50 \\
1,48 \\
1,58 \\
1,61 \\
1,76\end{array}$ \\
\hline
\end{tabular}

ceci durant 3 semaines. Le tableau 1 présente les facteurs d'allongement des temps de coagulation par rapport au même lait chauffé (temps de coagulation du lait cru/temps de coagulation du lait chauffé). Ils sont insignifiants à la dilution 8. D'après les autres valeurs, l'activité inhibitrice paraît être assez constante sur cet intervalle de temps.

Le calcul statistique appliqué à l'ensemble des résultats montre que pour chaque dilution enzymatique et pour chaque vache on obtient des coefficients de variation toujours compris entre 3 et $8 \%$ à une exception près. L'analyse de variance a fait ressortir que l'effet du temps est très faiblement significatif à $5 \%\left(F_{5,48}=2,7\right)$. 
TABLEAU 2 - TABLE 2

Effets des traitements thermiques du lait sur l'inhibition de la coagulation par la protéinase $\mathrm{MmR}$

Effects of thermal treatments of milk on the inhibition of coagulation by the $M m R$ proteinase

\begin{tabular}{|c|c|c|c|}
\hline & \multicolumn{2}{|c|}{ Traitement thermique } & \multirow{2}{*}{$\begin{array}{l}\text { Temps de coagulation } \\
\text { limite }^{*} \text { (min) }\end{array}$} \\
\hline & Durée & $\begin{array}{l}\text { Température } \\
\left({ }^{\circ} \mathrm{C}\right)\end{array}$ & \\
\hline Iait cru & 0 & - & 13 \\
\hline Lait chauffé (a) & $\begin{array}{l}20 \mathrm{~s} \\
20 \mathrm{~s} \\
20 \mathrm{~s} \\
20 \mathrm{~s}\end{array}$ & $\begin{array}{l}60 \\
65 \\
68 \\
72\end{array}$ & $\begin{array}{r}13 \\
20 \\
30 \\
\geqslant 60\end{array}$ \\
\hline Lait chauffé (b) & $\begin{array}{c}5 \mathrm{~min} \\
5 \mathrm{~min} \\
20 \mathrm{~s}\end{array}$ & $\begin{array}{l}55 \\
60 \\
72\end{array}$ & $\begin{aligned} & 25 \\
\geqslant & 60 \\
\geqslant & 60\end{aligned}$ \\
\hline Lait congelé (c) & $\begin{array}{l}1 \text { jour à } \\
3 \text { mois }\end{array}$ & -25 & 13 \\
\hline Lait lyophilisé & - & - & 13 \\
\hline
\end{tabular}

* Au-delà de ce temps, il n'y a plus proportionnalité entre le temps de coagulation et la dilution de l'enzyme.

\section{Effets de divers traitements du lait cru}

Nous avons répété et complété les expériences de Alais, Ducroo et Delecourt (1974) concernant l'effet du chauffage du lait cru sur l'activité inhibitrice vis-à-vis de la protéinase de Mucor miehei (MmR). Les résultats résumés dans le tableau 2 (parties a et b) confirment ceux des précédents auteurs; il est clair que l'inhibition disparaît dans des conditions assez douces, notamment dans celles de la pasteurisation HTST $\left(73^{\circ} \mathrm{C}, 25 \mathrm{~s}\right)$.

D'autres expériences ont été faites à basse température. La congélation à $-25^{\circ} \mathrm{C}$ avec conservation pendant des durées allant de quelques jours à plusieurs mois, ainsi que la lyophilisation, n'affectent pas l'activité inhibitrice, aussi bien dans le lait cru que dans le sérum d'ultracentrifugation (tab. 2, partie c). 


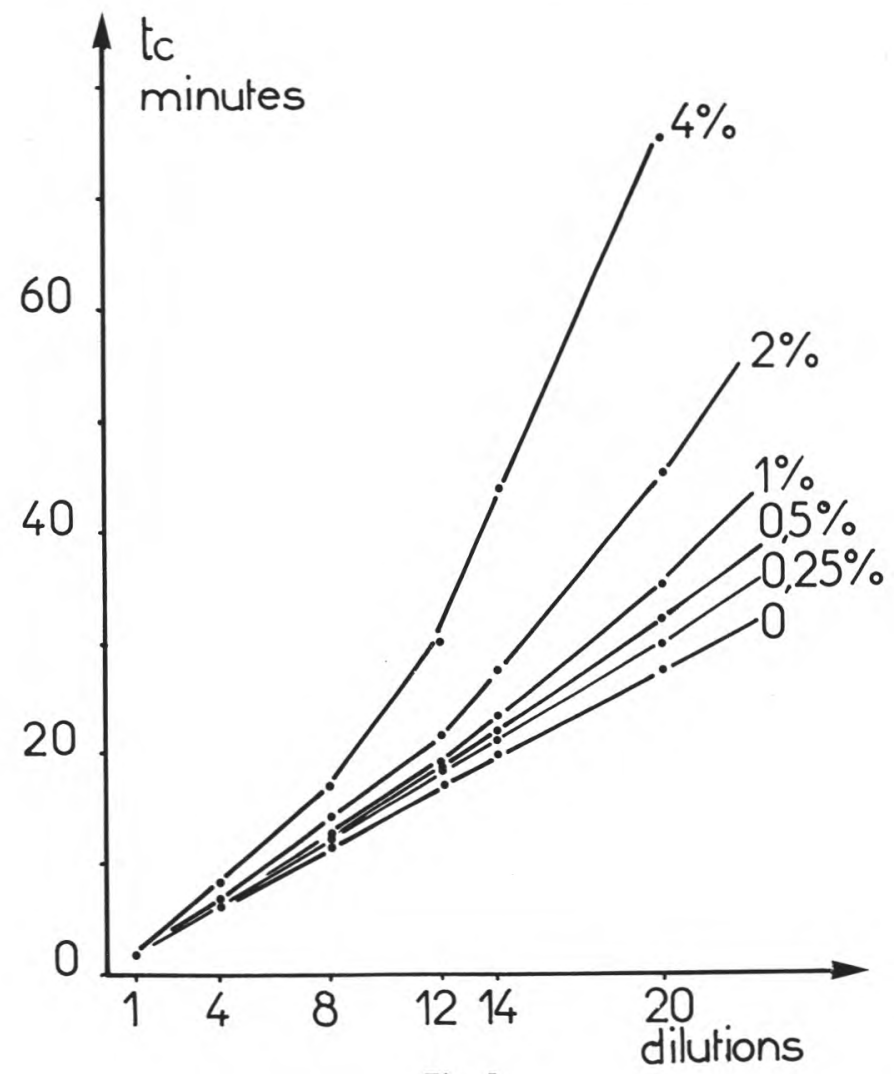

Fig. 5

Evolution du temps de coagulation d'un lait reconstitué standard additionné de quantités croissantes de poudre de lactosérum contenant la substance inhibitrice en fonction de la dilution de la protéinase MmR (dilution 1:0,8\%; tc : $3 \mathrm{~min}$ ).

Evolution of clotting-time of standard reconstitued milk containing different proportions of inhibitory whey with different dilutions of the MmR-proteinase (dilution 1: 0,8\%;Ct: $3 \mathrm{~min}$ ).

La dialyse prolongée contre l'eau n'influe pas sur cette activité. Il en va de même pour le traitement à $\mathrm{pH} \mathrm{4,6}$ : l'activité se retrouve dans le lactosérum isoélectrique.

L'homogénéisation sous haute pression (600 bars), avec un appareil Manton-Gaulin muni d'un clapet " Micro-gap » (Humbert et al., 1980), n'affecte pas l'activité inhibitrice contenue dans le sérum, après un simple passage. 


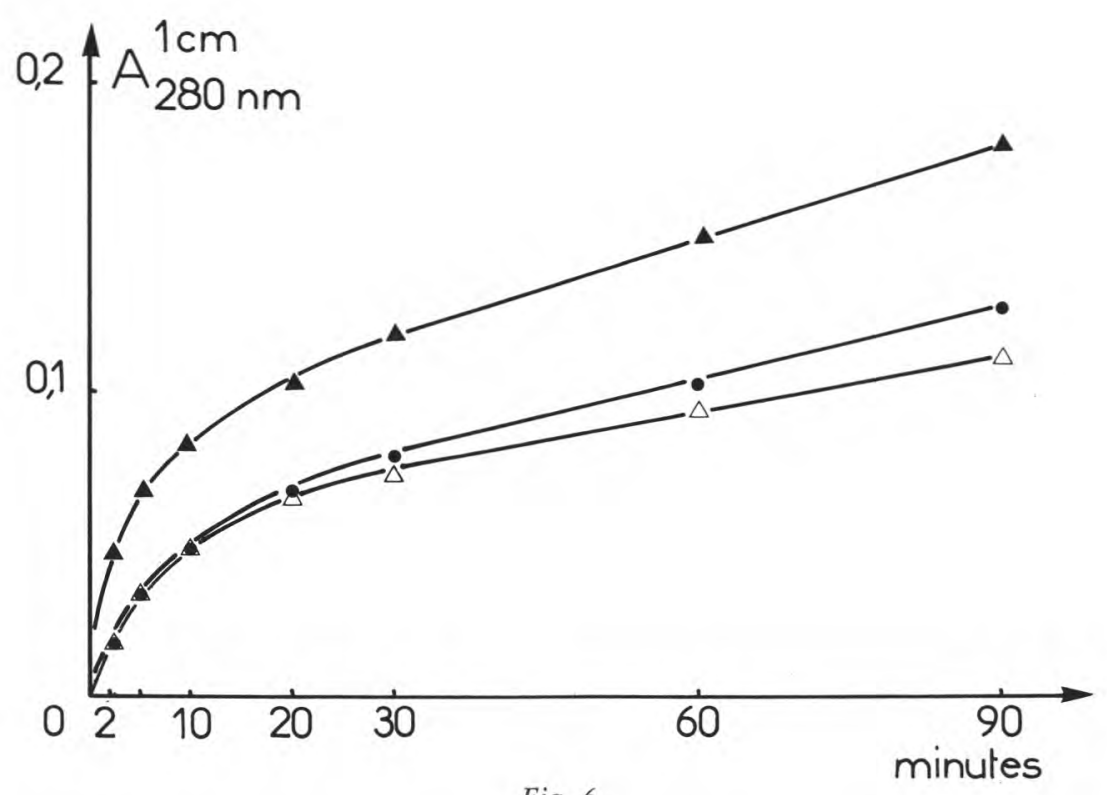

Fig. 6

Protéolyse d'une solution de caséine $(1 \%)$ additionnée de lactosérum inhibiteur (1 ou $2 \%)$ par la protéinase $\mathrm{MmR}\left(0,04 \%\right.$ à $\left.30^{\circ} \mathrm{C}, \mathrm{pH} 6,5\right)$.

- $1 \%$ de lactosérum " témoin " chauffé à $73^{\circ} \mathrm{C}, 25 \mathrm{~s}$.

- $1 \%$ de lactosérum non chauffé.

$\triangle \longrightarrow 2 \%$ de lactosérum non chauffé.

Proteolysis of $1 \%$ casein solution containing 1 or $2 \%$ inhibitory whey by the $M m R$ proteinase $\left(0,04 \%\right.$ at $\left.30^{\circ} \mathrm{C}, \mathrm{pH} 6,5\right)$.

$\triangle 1 \%$ of whey heated at $73^{\circ} \mathrm{C}, 25 \mathrm{~s}$.

$-11 \%$ of unheated whey.

$\triangle \longrightarrow \triangle 2 \%$ of unheated whey.

\section{Effets de l'addition d'albumines et de globulines}

Les protéines sont ajoutées à la concentration de $0,25 \%$ dans le lait reconstitué (non inhibiteur par lui-même) et l'on attend $1 \mathrm{~h}$ pour faire l'épreuve de coagulation avec différentes dilutions de l'enzyme MmR.

Dans ces conditions, aucune inhibition n'est perceptible avec la sérum-albumine et la $\beta$-lactoglobuline isolées du lait de vache. Par contre, une faible inhibition se manifeste avec les immunoglobulines sanguines bovines à de fortes dilutions de l'enzyme.

6. Relation entre la concentration de l'inhibiteur et le temps de coagulation

On a séché par lyophilisation le lactosérum d'un lait présentant une forte activité inhibitrice envers les enzymes de Mucor miehei. 


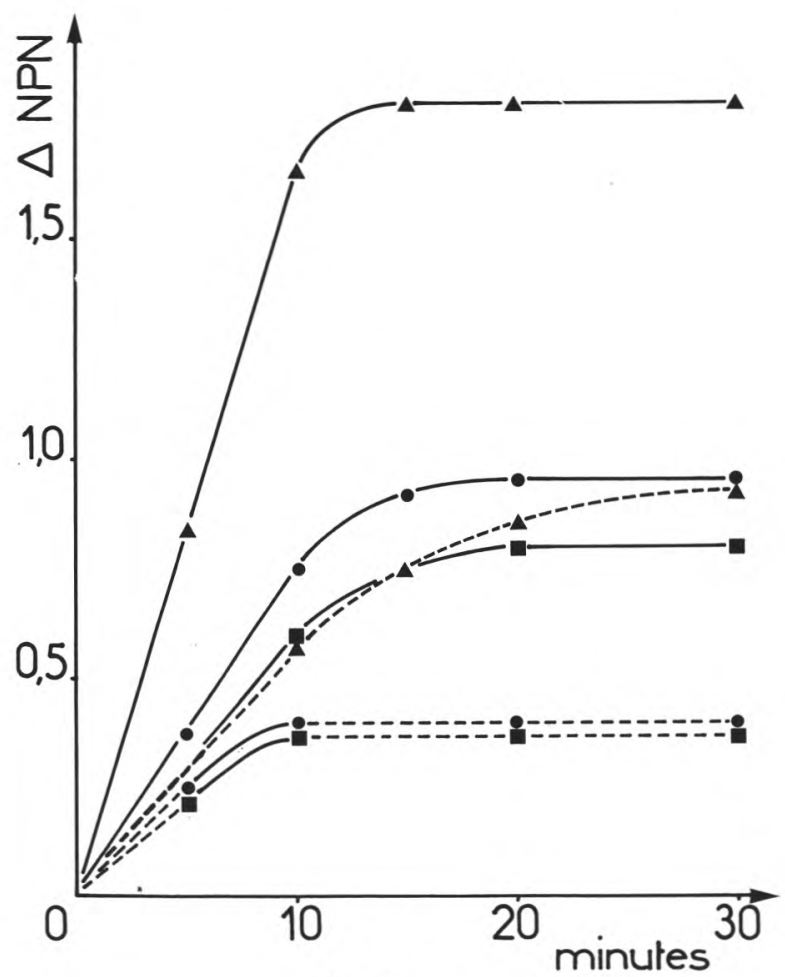

Fig. 7

Protéolyse du lait de vache et de chèvre par la protéinase $\mathrm{MmR}$ $\left(30^{\circ} \mathrm{C}, \mathrm{pH} 6,5\right)$.

Lait de vache cru $\mathbf{-}-\mathbf{\Lambda}$ et chauffé $\mathbf{\Delta}$ (MmR 0,005\%)

Lait de vache cru $\bullet-\ldots$ et chauffé $-\longrightarrow(\mathrm{MmR} \mathrm{0,01 \% )}$

Lait de chèvre cru - - - et chauffé - (MmR 0,008\%)

(Le lait est chauffé à $73^{\circ} \mathrm{C}, 25 \mathrm{~s}$ ).

Proteolysis in bovine and goat milk by the MmR-proteinase $\left(30^{\circ} \mathrm{C}\right.$, pH 6,5).

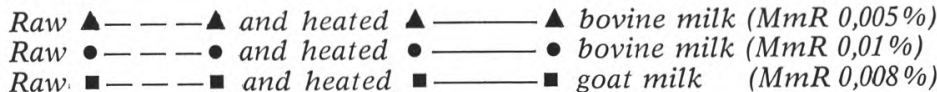

(The milk is heated at $73^{\circ} \mathrm{C}, 25 \mathrm{~s}$ ).

Ce lactosérum sec a été ajouté à des concentrations allant de 0,25 à $4 \%$, à du lait reconstitué.

Les courbes de la figure 5 montrent que l'inhibition est approximativement proportionnelle à la quantité d'inhibiteur pour des dilutions supérieures à 12 . 


\section{Inhibition de l'activité protéolytique}

\section{Dans une solution de caséine entière}

La figure 6 présente les courbes de protéolyse par l'enzyme $\mathrm{MmR}$ pendant une durée de 2 à $90 \mathrm{~min}$. Elles montrent une diminution significative de la libération de l'azote non protéique quand le substrat contient $1 \%$ de lactosérum cru; l'inhibition est encore plus grande lorsque l'on double la dose de lactosérum. Il est à noter que $1 \mathrm{~g}$ de lactosérum dialysé sec correspond à environ $80 \mathrm{ml}$ de lactosérum brut.

\section{Dans le lait}

Les courbes de la figure 7 montrent que la protéolyse par l'enzyme $M m R$ est largement freinée dans le lait cru par rapport au même lait préalablement chauffé $\left(73^{\circ} \mathrm{C}, 25 \mathrm{~s}\right)$. Après 15 à $20 \mathrm{~min}$ de digestion, la proportion de NPN libéré passe du simple au double. L'effet d'inhibition sur la protéolyse est donc très net.

\section{DISCUSSION}

L'inhibition par le lait cru de l'activité de protéinases coagulantes fongiques n'est pas spécifique. Déjà observée dans le lait de vache (Alais, Ducroo et Delecourt, 1974) elle a été retrouvée dans le lait de brebis et de chèvre.

Les protéinases acides des Mucor (miehei, pusillus) sont sensibles à cette inhibition; alors que d'autres enzymes coagulantes appartenant à la même famille des « aspartates protéinases » (Hofmann, 1974) ne subissent pas cette inhibition : protéinases gastriques (pepsine, chymosine) et protéinase de Endothia parasitica. Malgré les analogies de structure primaire et spatiale dans cette famille de protéinases et un mécanisme d'action probablement commun (Foltmann, 1981), la sensibilité aux inhibiteurs peut être différente d'une enzyme à l'autre.

Dans le lait cru, le degré d'inhibition varie d'une vache à l'autre. Ceci n'est pas lié à des différences dans l'aptitude à la coagulation des laits individuels, qui sont dues principalement aux variations de la teneur en calcium ionisé; ces différences se traduisent par un changement de pente de la droite reliant le temps de coagulation à l'inverse de la concentration en enzyme (dilution). Dans le phénomène étudié ici, la courbe cesse d'être une droite à partir d'une certaine dilution. On peut donc penser que la concentration en substance inhibitrice varie d'un lait à l'autre; par contre, elle varie peu d'un jour à l'autre, chez un individu en pleine période de lactation.

L'inhibition concomittante de l'activité protéolytique et de l'activité coagulante dans le lait cru montre une fois de plus la liaison 
de cause à effet, qui fut autrefois discutée, entre protéolyse limitée de la caséine (réaction primaire) et coagulation proprement dite (phase secondaire).

Le ou les inhibiteurs du lait cru sont facilement inactivés par le chauffage, comme cela découlait de la précédente étude (Alais, Ducroo et Delecourt, 1974). L'inhibiteur est insensible aux basses températures; on peut conserver le lait ou le lactosérum à l'état congelé ou lyophilisé sans perte de l'activité antiprotéinasique.

La dénaturation thermique et la rétention au cours de la dialyse suggèrent que nous avons probablement à faire à un inhibiteur de nature protéique. Des essais en cours indiquent qu'il peut être séparé par les méthodes de fractionnement des protéines du sérum. L'inhibition est donc différente de celle de l'enzyme coagulante de Bacillus subtilis, étudiée par Dutta et al. (1971) qui est due à de petites molécules comme la cystéine.

L'addition d'immunoglobulines sanguines bovines a un faible effet inhibiteur. Les protéines du lactosérum de bas poids moléculaire sont sans effet. On a précédemment discuté (Alais, Ducroo, Delecourt, 1974), une théorie selon laquelle les globulines retarderaient la coagulation enzymatique par un effet de " colloïde protecteur "(Puhan 1967) et qui ne semble pas satisfaisante pour une inhibition dirigée seulement contre des protéinases de Mucor. L'inhibition ne concerne qu'un type de protéinases; on peut envisager que l'inhibiteur soit un anticorps passant dans le lait, à la suite de l'immunisation contre des micro-organismes qui appartiennent à la microflore banale du sol.

Reçu pour publication en mai 1982.

\section{Remerciements}

Nous remercions G. Linden et J. Brun-Bellut pour leurs conseils dans la réalisation de ce travail.

\section{Bibliographie}

Alais (C.) et NovaK (G.) (1968). - Etude d'une enzyme coagulante microbienne dérivée de Endothia parasitica. Lait, 48, 393-418.

Alais (C.), Ducroo (P.) et Delecourt (R.) (1974). - Inhibition d'une protéase coagulante fongique dans le lait cru. Lait, 54, 517-527.

BERRIDGE (N. J.) (1945). - The purification and cristallisation of rennin. Biochem. J. 39, 179-185.

BRIOT (A.) (1900). - Etudes sur la présure et l'antiprésure. Thèse Docteur èsSciences Naturelles, Paris. Série A, $\mathrm{N}^{\circ} 361, \mathrm{n}^{\circ}$ d'ordre 1027.

Dutta (S. M.), Kuila (R. K.), SRinivasan (R. A.), Babbar (I. J.) and Dudani (A. T.) (1971). - Milk clotting enzymes from spore forming bacterial isolates. Milchwissenschaft, 26, 683-685. 
FoltManN (B.) (1981). - Mammalian milk-clotting proteases: Structure, function evolution, development. Neth. Milk Dairy J., 35, 223-231.

Hofmann (T.) (1974). - Structure, function and evolution of acid proteases, in Advances in Chemistry Series $\mathrm{n}^{\circ} 136$, edited by the American Society.

Humbert (G.), Driou (A.), GuéRin (J.) et Alais (C.) (1980). - Etude de l'homogénéisation à haute pression sur les propriétés du lait et son aptitude à la coagulation enzymatique. Lait, 60, 574-594.

Puhan (Z.) (1966). - Die Anwendung einer Bacillus subtilis Protease als Labersatzstoff. Dissertation, Zurich $\mathrm{n}^{\circ} 3861$.

Puhan (Z.) (1967). - Einfluß der Immunoglobuline auf die Gerinnung der Milch mit mikrobiellen Labersatzstoff. Milchwissenschaft, 22, 133-138.

Ramet (J. P.) et Alais (C.) (1973). - Etude d'une protéase coagulante produite par Mucor miehei. Lait, 53, 154-162.

Singh (L. N.) et Ganguli (N. C.) (1973). - Inhibition of rennin activity by bovine blood serum. Milchwissenschaft, 28, 565-568.

Tsugo (T.) and Yamauchi (K.) (1953). - The inhibition of milk coagulating enzymes by blood serum. 13e Congrès International de Laiterie, La Haye, vol. 4, 641-644. 